\title{
Correlation between optic disc deformation and retinal vasculature in non-pathological high myopia
}

\author{
JIAO SUN, JIALIN WANG and YANLING WANG \\ Department of Ophthalmology, Beijing Friendship Hospital \\ Affiliated to Capital Medical University, Beijing 100050, P.R. China
}

Received January 23, 2020; Accepted August 13, 2020

DOI: $10.3892 /$ etm.2021.9811

\begin{abstract}
The aim of the current study was to investigate the correlation between optic disc deformation and retinal vasculature in high myopia. A total of 130 eyes with non-pathological high myopia were included in the current cross-sectional study. $\beta$-zone parapapillary atrophy ( $\beta$-PPA), optic disc tilt ratio, and horizontal and vertical disc diameters were analyzed using fundus color photography. A 3x3 mm grid and a $4.5 \times 4.5 \mathrm{~mm}$ grid were used to scan parafoveal and peripapillary regions, respectively, using optical coherence tomography angiography. Vessel flow density (VFD) and fractal dimension of the retina, as well as the foveal avascular zone (FAZ), were analyzed and quantified using en face projection images. Optic disc parameters that were associated with vascular changes were determined using multiple linear regression analysis. The results from the multivariate analysis revealed that $\beta$-PPA was negatively correlated with the VFD of the superficial retinal plexus $(\mathrm{R}=-2.805 ; \mathrm{P}=0.006)$, deep retinal plexus $(\mathrm{R}=-2.801 ; \mathrm{P}=0.006)$, radial parapapillary capillaries $(\mathrm{R}=-3.936 ; \mathrm{P}<0.001)$ and enhanced-depth imaging of the fovea $(\mathrm{R}=-2.161 ; \mathrm{P}=0.034)$. Additionally, FAZ was not significantly correlated with any factors in the current study. Age was negatively correlated with the VFD of the retina $(\mathrm{R}=-4.234 ; \mathrm{P}<0.001)$, while the optic tilt ratio $(\mathrm{R}=-2.291$; $\mathrm{P}=0.025)$ was negatively correlated with three sectors in the deep layer. Overall, the present results demonstrated that optic disc deformation was negatively correlated with the retinal microvasculature in non-pathological high myopia,
\end{abstract}

Correspondence to: Dr Jialin Wang, Department of Ophthalmology, Beijing Friendship Hospital Affiliated to Capital Medical University, 95 Yong'an Road, Beijing 100050, P.R. China E-mail: wangjialin@bjmu.edu.cn

Abbreviations: $\beta$-PPA, $\beta$-zone parapapillary atrophy; DRP, deep retinal plexus; FAZ, foveal avascular zone; IPL, inner plexiform layer; OCTA, optical coherence tomography angiography; RPC, radial peripapillary capillary; SRP, superficial retinal plexus

Key words: $\beta$-zone parapapillary atrophy, tilt ratio, high myopia, optical coherence angiography particularly in the radial peripapillary capillaries and the deep retinal plexus. Therefore, optic disc deformation may be used to predict the retinal vasculature in high myopia.

\section{Introduction}

Myopia is one of the most commonly reported ocular disorders worldwide, and there has recently been a significant increase in the myopic population in China (1). The costs of examinations and surgical corrections of myopia are significant, and this disorder has been associated with other pathological eye conditions. High myopia is associated with reduced retinal perfusion. In fundus photography, retinal vessel density and blood flow are markedly reduced in highly myopic eyes (2). Using a dynamic vessel analyzer, it was previously demonstrated that there was a narrowing of retinal large vessels in high myopia (3). Since the retinal microvasculature directly supplies $\mathrm{O}_{2}$ and nutrients to the retinal tissues, this vasculature is more susceptible to myopia-related alterations (4). Understanding these changes to the retinal large vessels and microvasculature will ultimately aid in the early diagnosis and monitoring of retinopathy in patients with high myopia. However, previous studies are limited due to the imaging modalities used. For instance, only large vessels could be imaged, and invasive methods were required, such as fluorescein angiography (5). These imaging modalities may prevent researchers from investigating alterations to the retinal microvasculature as early indicators of the onset of retinopathy. However, with the recent advances in optical coherence tomography angiography (OCTA), the vasculature can be measured quantitatively in a noninvasive manner in terms of morphological information, which can provide depth-resolved visualization of the retinal microvasculature (6).

Morphological changes to the optic disc in myopic eyes are induced by posterior scleral remodeling during axial elongation, including $\beta$-zone parapapillary atrophy ( $\beta$-PPA), optic disc tilt and optic disc rotation $(7,8)$. Since the optic disc becomes smaller as myopia progresses (9), it was hypothesized that myopic deformation of the optic disc may be associated with macular and disc perfusion. Therefore, the purpose of the current study was to determine the correlation between optic disc deformation and retinal vasculature in non-pathological high myopia using OCTA imaging. 


\section{Materials and methods}

Participants. The current study was approved by the Beijing Friendship Hospital Affiliated to Capital Medical University (Beijing, China), and was conducted in accordance with the ethical standards stated in the Declaration of Helsinki and the Health Insurance Portability and Accountability Act. Written informed consent was obtained from all the examined patients and volunteers participating in the study prior to OCTA imaging. Patients from the Beijing Friendship Hospital (Beijing, China) were recruited from April 2018 to September 2018. A total of 77 patients were included in the study. The mean age was $35.24 \pm 8.45$ years (age range, $20-55$ ). Subjects comprised 37 males and 40 females.

Each patient underwent a complete ocular examination that included best-corrected visual acuity testing, intraocular pressure (IOP) evaluations using an automatic tonometer, slit-lamp examinations, funduscopy and axial length (AL) measurements using optical biometry (IOLMaster ${ }^{\circledR}$; Carl Zeiss AG).

A total of 130 eyes with non-pathological high myopia were included in the current cross-sectional study. Patients with high myopia and a refraction of <- 6 diopters or ALs $>26.5 \mathrm{~mm}$ were included in the current study. Furthermore, patients with a history of prior vitreous or retinal surgery, an IOP of $>21 \mathrm{mmHg}$ or evidence of retinal disease (other than myopic degeneration) that affected the retinal or choroidal vasculature as evidenced by history or examination were excluded. Additionally, eyes that exhibited diffuse retinal pigment epitheliopathy (RPE) atrophy due to high myopia or any structural changes, including myopic choroidal neovascularization, were excluded from the analyses.

Image acquisition and analysis. OCTA imaging was performed using an RTVue XR Avanti system with AngioVue version 2016.2.0.35 (Optovue, Inc.) at a scanning speed of 70,000 A-scans/sec. All imaging was performed by a single operator (JS). The scan protocol examined a $3.0 \times 3.0-\mathrm{mm}^{2}$ area focused on the macula and a $4.5 \times 4.5-\mathrm{mm}^{2}$ area focused on the optic disc. Vessel flow density (VFD) and fractal dimension of the retina, as well as the foveal avascular zone (FAZ), were analyzed and quantified using en face projection images. Enhanced-depth imaging of the fovea was also acquired by OCTA (Fig. 1). VFD was measured at the SRP and DRP in the macular region. Each layer was divided into four sectors: i) Nasal; ii) temporal; iii) superior and iv) inferior. The perfusion of the optic disc was divided into three layers: i) Optic disc head (ONH); ii) radial peripapillary capillary (RPC) and iii) choroid. Horizontal and vertical priorities in $\sim 2.9 \mathrm{sec}$ for each of the two raster scans were obtained. The superficial retinal plexus (SRP) was segmented from the outer boundary of the inner limiting membrane (ILM) to the outer boundary of the inner plexiform layer (IPL), which extends from $3 \mu \mathrm{m}$ below the ILM to $15 \mu \mathrm{m}$ below the IPL. The deep retinal plexus (DRP) was segmented from the outer boundary of the IPL to the outer boundary of the outer plexiform layer, which extends from 15 to $70 \mu \mathrm{m}$ below the IPL.

Since magnification is different in myopic eyes, the imaging sampling density used in myopic eyes must be lower than that used in normal eyes. Therefore, the magnification of the obtained images was corrected for highly myopic eyes using Bennett's formula (10). Two independent examiners (JS and JW) reviewed each image. Poor-quality images were excluded based on the following criteria: i) Evidence of poor fixation, including a double vessel pattern and motion artifacts; ii) presence of motion artifacts that could not be corrected by motion correction technology; iii) media opacity, as exhibited by shadowing or obscuration of the vessel signal in the field of view or a signal strength index of $<40$; and iv) segmentation error in the defining vascular layers.

OCTA data were analyzed using Optovue software (version no. 2016.2.0.35; Optovue, Inc.). The foveal avascular zone (FAZ) area for each superficial plexus image was determined and measured using the built-in non-flow automatic measurement tool of the AngioVue review software version 2016.2.0.35 (Optovue, Inc.).

High-resolution digital color fundus photographs were obtained using a digital retina camera (Kowa Nonmyd WX; Kowa Company, Ltd.). Image processing of the $\beta$-PPA area, optic disc tilt ratio, and horizontal and vertical optic disc diameter measurements were performed using the public domain ImageJ software (version no. 1.50i; National Institutes of Health). Two examiners (JS and JW) measured each image three times to assess the reproducibility of the technique.

Statistical analysis. Statistical analysis was performed using a commercially available statistical software program (SPSS for Microsoft; version 24.0; IBM Corp.). Firstly, the mean and standard deviation of the main outcome parameters were calculated. Following this, a regression analysis using the angiographic parameters as the dependent variables was performed. The parameters that were significantly associated with the angiographic parameters following the univariate analysis were used as independent variables. For all analyses, $\mathrm{P}<0.05$ was considered to indicate a statistically significant difference.

\section{Results}

Demographics. The demographic and ocular characteristics of the participants are presented in Table I. A total of 130 eyes from 77 participants with nonpathological high myopia were analysed in this study. The mean age was $35.24 \pm 8.45$ years, and the mean spherical equivalent (SE) refractive error was $-10.03 \pm 3.57 \mathrm{D}$. The demographics of these participants are shown in Table I.

Association between optic disc deformation and macular perfusion parameters. Vessel flow density was measured at the SRP and DRP in the macular region. Each layer was divided into four sectors: i) Nasal; ii) temporal; iii) superior; and iv) inferior. The results from the regression analysis revealed that each sector of the macular superficial layer was negatively correlated with $\beta$-PPA (mean, $\mathrm{R}=-2.805 ; \mathrm{P}=0.006$; Table II), while the nasal sector was negatively correlated with age $(\mathrm{R}=-2.116 ; \mathrm{P}=0.038)$. Furthermore, FAZ was not correlated with age, optic tilt ratio, horizontal optic disc diameter and $\beta$-PPA in the current study.

Table III presents the correlations between optic disc deformation and DRP parameters. There was a negative correlation between $\beta$-PPA and the mean $(R=-2.801$; 
Table I. Demographic and ocular characteristics of the participants.

\begin{tabular}{|c|c|c|c|}
\hline Characteristic & Mean \pm SD $(n=77)$ & Median $(n=77)$ & Range $(n=77)$ \\
\hline Age (years) & $35.24 \pm 8.45$ & 34.50 & $20.00-55.00$ \\
\hline \multicolumn{4}{|l|}{ Sex } \\
\hline Male & 37 & & \\
\hline Female & 40 & & \\
\hline SE (D) & $10.03 \pm 3.57$ & 8.94 & $6.15-20.13$ \\
\hline $\mathrm{AL}(\mathrm{mm})$ & $27.43 \pm 1.68$ & 27.28 & $26.10-34.46$ \\
\hline $\mathrm{SP}(\mathrm{mmHg})$ & $119.95 \pm 11.90$ & 122.00 & $91.00-149.00$ \\
\hline $\mathrm{DP}(\mathrm{mmHg})$ & $74.67 \pm 8.75$ & 75.00 & $56.00-103.00$ \\
\hline HR (bpm) & $76.11 \pm 8.33$ & 76.00 & $59.00-93.00$ \\
\hline IOP (mmHg) & $15.62 \pm 3.25$ & 15.15 & $9.90-20.80$ \\
\hline Optic tilt ratio & $1.29 \pm 0.18$ & 1.26 & $0.91-1.76$ \\
\hline Horizontal optic disc diameter (mm) & $1.33 \pm 0.17$ & 1.33 & $1.01-2.04$ \\
\hline Vertical optic disc diameter (mm) & $1.05 \pm 0.19$ & 1.05 & $0.67-1.57$ \\
\hline$\beta$-PPA $\left(\mathrm{mm}^{2}\right)$ & $1.09 \pm 0.62$ & 0.88 & $0.19-2.85$ \\
\hline RNFL $(\mu \mathrm{m})$ & $95.69 \pm 9.49$ & 95.00 & $72.00-117.00$ \\
\hline $\mathrm{C} / \mathrm{D}$ & $0.28 \pm 0.18$ & 0.28 & $0.03-0.65$ \\
\hline Disc area $\left(\mathrm{mm}^{2}\right)$ & $2.01 \pm 0.59$ & 1.93 & $0.94-4.38$ \\
\hline
\end{tabular}

SE, spherical equivalent; D, diopters; AL, axial length; SP, systolic pressure; DP, diastolic pressure; HR, heart rate; IOP, intraocular pressure; $\beta$-PPA, $\beta$-peripapillary atrophy; RNFL, retinal nerve fiber layer; $\mathrm{C} / \mathrm{D}$, cup-to-disc area ratio.

A

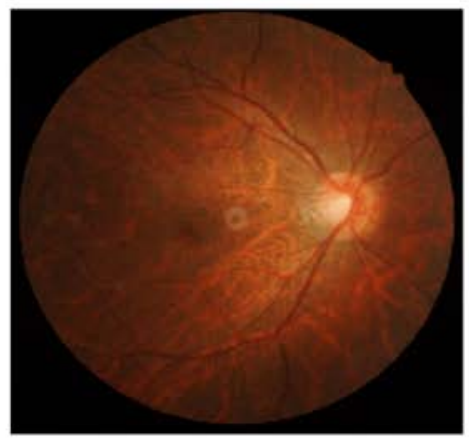

D

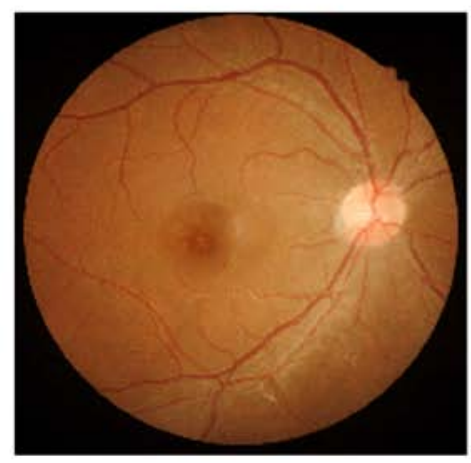

B

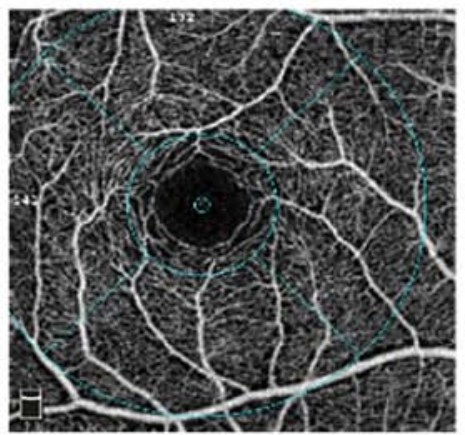

E

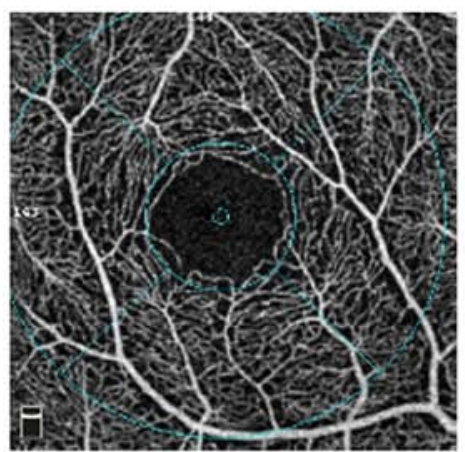

C

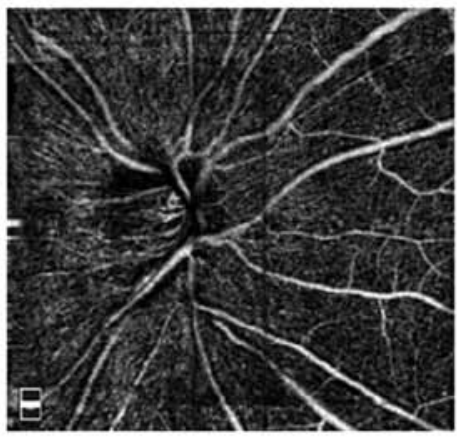

F

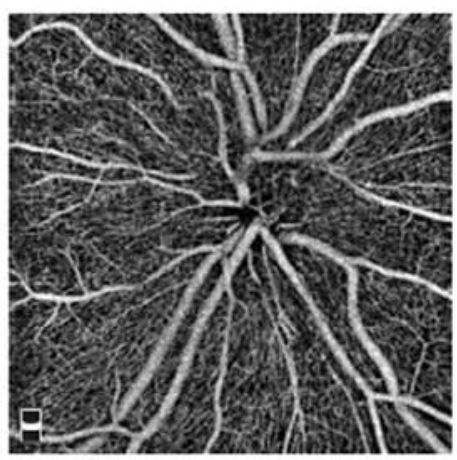

Figure 1. Image of healthy volunteer and high myopia with tessellated fundus and optic disc deformation. (A) Color fundus photograph, (B) foveal avascular zone and vessel image and (C) image of the radial peripapillary capillaries in the healthy volunteer. (D) Color fundus photograph, (E) foveal avascular zone and vessel image and $(\mathrm{F})$ image of the radial peripapillary capillaries in a patient with high myopia.

$\mathrm{P}=0.006)$, nasal $(\mathrm{R}=-2.743 ; \mathrm{P}=0.008)$ and temporal sectors $(\mathrm{R}=-2.740 ; \mathrm{P}=0.008)$. Additionally, there was a negative correlation between optic disc tilt and DRP perfusion parameters in the mean $(\mathrm{R}=-2.291 ; \mathrm{P}=0.025)$ and inferior
$(\mathrm{R}=-3.667 ; \mathrm{P}<0.001)$ regions. Furthermore, the horizontal optic disc diameter was negatively correlated with superior retinal vessel density $(\mathrm{R}=-1.995 ; \mathrm{P}=0.050)$. Table III presents a negative association between age and the mean $(\mathrm{R}=-2.429$; 
Table II. Correlation between age, optic disc deformation, superficial retinal plexus perfusion parameters and FAZ.

\begin{tabular}{|c|c|c|c|c|c|c|c|c|c|c|c|c|}
\hline \multirow[b]{2}{*}{ Parameter } & \multicolumn{2}{|c|}{ Mean } & \multicolumn{2}{|c|}{ Temporal } & \multicolumn{2}{|c|}{ Superior } & \multicolumn{2}{|c|}{ Nasal } & \multicolumn{2}{|c|}{ Inferior } & \multicolumn{2}{|c|}{ FAZ } \\
\hline & $\mathrm{R}$ & P-value & $\mathrm{R}$ & $\mathrm{P}$-value & $\mathrm{R}$ & P-value & $\mathrm{R}$ & P-value & $\mathrm{R}$ & P-value & $\mathrm{R}$ & P-value \\
\hline Age (years) & -1.271 & 0.207 & -1.064 & 0.291 & -0.702 & 0.485 & -2.116 & 0.038 & -1.672 & 0.099 & 1.020 & 0.311 \\
\hline Optic tilt ratio & -0.365 & 0.723 & -0.377 & 0.707 & -0.431 & 0.668 & 0.336 & 0.738 & -0.750 & 0.456 & -0.592 & 0.556 \\
\hline $\begin{array}{l}\text { Horizontal optic } \\
\text { disc diameter (mm) }\end{array}$ & 0.134 & 0.894 & 0.162 & 0.871 & 0.559 & 0.578 & 0.724 & 0.472 & 0.245 & 0.807 & 0.879 & 0.382 \\
\hline $\mathrm{AL}(\mathrm{mm})$ & -0.307 & 0.001 & -0.346 & $<0.001$ & -0.248 & 0.006 & -0.331 & $<0.001$ & -0.315 & $<0.001$ & -0.252 & 0.006 \\
\hline$\beta-\mathrm{PPA}$ & -2.805 & 0.006 & -2.337 & 0.022 & -2.130 & 0.036 & -2.865 & 0.005 & -2.958 & 0.004 & -0.002 & 0.998 \\
\hline
\end{tabular}

FAZ, foveal avascular zone; AL, axial length; $\beta$-PPA, $\beta$-peripapillary atrophy.

Table III. Correlation between age, optic disc deformation and deep retinal plexus perfusion parameters.

\begin{tabular}{|c|c|c|c|c|c|c|c|c|c|c|}
\hline \multirow[b]{2}{*}{ Parameter } & \multicolumn{2}{|c|}{ Mean } & \multicolumn{2}{|c|}{ Temporal } & \multicolumn{2}{|c|}{ Superior } & \multicolumn{2}{|c|}{ Nasal } & \multicolumn{2}{|c|}{ Inferior } \\
\hline & $\mathrm{R}$ & P-value & $\mathrm{R}$ & P-value & $\mathrm{R}$ & P-value & $\mathrm{R}$ & $\mathrm{P}$-value & $\mathrm{R}$ & P-value \\
\hline Age & -2.429 & 0.017 & -1.630 & 0.107 & -0.695 & 0.489 & -2.341 & 0.022 & -3.883 & $<0.001$ \\
\hline Optic tilt ratio & -2.291 & 0.025 & -1.512 & 0.135 & -0.229 & 0.819 & -1.910 & 0.060 & -3.667 & $<0.001$ \\
\hline $\begin{array}{l}\text { Horizontal optic } \\
\text { disc diameter (mm) }\end{array}$ & -1.065 & 0.290 & -1.344 & 0.183 & -1.995 & 0.050 & -0.765 & 0.447 & 1.298 & 0.198 \\
\hline $\mathrm{AL}(\mathrm{mm})$ & -0.400 & $<0.001$ & -0.275 & 0.002 & -0.404 & $<0.001$ & -0.307 & 0.001 & -0.400 & $<0.001$ \\
\hline$\beta$-PPA & -2.801 & 0.006 & -2.740 & 0.008 & -0.264 & 0.792 & -2.743 & 0.008 & -1.489 & 0.141 \\
\hline
\end{tabular}

AL, axial length; $\beta$-PPA, $\beta$-peripapillary atrophy.

Table IV. Correlation between optic disc deformation, perfusion parameters and subfoveal choroidal thickness.

\begin{tabular}{|c|c|c|c|c|}
\hline \multirow[b]{2}{*}{ Parameter } & \multicolumn{2}{|c|}{$\mathrm{RPC}$} & \multicolumn{2}{|c|}{$\begin{array}{l}\text { Subfoveal choroidal } \\
\text { thickness }(\mu \mathrm{m})\end{array}$} \\
\hline & $\mathrm{R}$ & P-value & $\mathrm{R}$ & P-value \\
\hline Age (years) & -1.656 & 0.102 & -4.234 & $<0.001$ \\
\hline Optic tilt ratio & -1.870 & 0.065 & -1.797 & 0.076 \\
\hline Horizontal optic disc diameter (mm) & 0.139 & 0.890 & -2.281 & 0.025 \\
\hline $\mathrm{AL}(\mathrm{mm})$ & -0.053 & 0.175 & -0.431 & $<0.001$ \\
\hline$\beta$-PPA & -3.936 & $<0.001$ & -2.161 & 0.034 \\
\hline
\end{tabular}

RPC, radial peripapillary capillary; AL, axial length; $\beta$-PPA, $\beta$-peripapillary atrophy.

$\mathrm{P}=0.017)$, nasal $(\mathrm{R}=-2.341 ; \mathrm{P}=0.022)$ and inferior $(\mathrm{R}=-3.883$; $\mathrm{P}<0.001)$ regions.

Association between optic disc deformation and perfusion parameters. The results of the regression analysis between optic disc deformation and optic disc perfusion parameters are presented in Table IV. The perfusion of the optic disc was divided into three layers: i) Optic disc head (ONH); ii) radial peripapillary capillary (RPC); and iii) choroid. The RPC was negatively correlated with $\beta$-PPA $(\mathrm{R}=-3.936$; $\mathrm{P}<0.001)$, while the subfoveal choroidal thickness was negatively correlated with age $(\mathrm{R}=-4.234 ; \mathrm{P}<0.001), \beta-\mathrm{PPA}(\mathrm{R}=-2.161 ; \mathrm{P}=0.034)$ and horizontal optic disc diameter $(\mathrm{R}=-2.281 ; \mathrm{P}=0.025)$.

\section{Discussion}

The current study demonstrated that there were significant correlations between optic disc deformation and vascular parameters, including the vessel density of SRP, DRP and RPC. Compared to a previous report (11), the present study 
recruited participants whose SE are $-10.03 \pm 3.57 \mathrm{D}$ and performed regression analysis separately in each direction (superior, inferior, nasal and temporal).

The results demonstrated that the optic disc tilt ratio was correlated with the mean and inferior vessel density of the DRP. Furthermore, since disc tilt and torsion were significantly more frequent in the inferior direction, it is possible that changes in optic disc morphology may be associated with changes in inferior scleral thinning (12). Since it is difficult to precisely measure the true horizontal diameter of the optic disc, previous study has evaluated the amount of tilt by calculating the ratio between the minimum and maximum diameters of the nerve, a value termed the index of tilt (13).

In the current study, the superior vessel density of the DRP became lower as the horizontal disc diameter increased. However, Dai et al (14) reported that $\beta$-PPA and $\gamma$-PPA were associated with vertical disc diameter, and that the associations between $\beta$-PPA or $\gamma$-PPA and horizontal disc diameter were unclear and not significant. By contrast, Guo et al (15) demonstrated that the horizontal and vertical disc diameters were positively associated with the enlargement of $\gamma$-PPA.

The results of the present study revealed that vessel density in the RPC was negatively correlated with $\beta$-PPA. However, Fan et al (5) demonstrated that there were no differences in vascular density in the optic disc region among the three groups (control, moderate and high myopia), and vascular density in the optic disc region was not associated with AL, spherical equivalent or RNFL thickness.

Furthermore, the current study demonstrated that FAZ was not correlated with optic disc deformations. Wang et al (6) did not identify any differences in the area and diameter of the FAZ in healthy Chinese volunteers. This finding may indicate that the FAZ is not a suitable outcome to study changes in the microvessel network density of myopic eyes. Notably, the FAZ area did not significantly change in response to hyperoxia. However, most of the $\mathrm{O}_{2}$ supplied to the retina from the FAZ area is derived from the choroidal vessel, rather than from the retinal circulation, which may explain the lack of changes in the FAZ area in response to hyperoxia (16).

Garg et al (17) reported that choroidal thinning was associated with $\beta$-PPA. The current study demonstrated that subfoveal choroidal thickness was thinner in eyes with higher $\beta$-PPA than that in eyes with lower $\beta$-PPA. Furthermore, Wang et al (6) revealed that the density of the macular vascular networks in superior and deep layers and the choriocapillaris decreased with age.

$\beta$-PPA is associated with myopic eyeball axial elongation and temporal pulling of the optic nerve. The adjacent retinal tissue extends externally, and this mechanical stretching results in morphological changes in vessel and tissue thickness (18). During $\beta$-PPA, the shape of vessels becomes straighter and thinner, which may affect the vessel flow in the macular region (19). Furthermore, changes in vessel thickness may damage endothelial cells and subsequently reduce the concentration of VEGF $(20,21)$. Chui et al (22) revealed that retinal stretching may not mirror scleral growth, and that there is a difference between the photoreceptor margin and RPE margin in certain eyes, indicating that slippage may occur during eye growth within the retina. This may result in retinoschisis and subsequently reduce perfusion in the macula.

There is interplay between genetic factors and environmental stressors in the development of myopia. Myopia is typically exhibited with apparent familial aggregation; however, genetic factors alone cannot explain the rapid increase in the prevalence of myopia over the past one or two generations (23). Bredrup et al (24) reported that the clinical characteristics of a family with a distinctly excavated optic disc anomaly exhibited optic nerve dysplasia, high-grade myopia and increased ALs. Additionally, genetic analysis revealed a co-segregation of this optic disc anomaly with a mutation in the MYC-binding protein 2 gene. Overall, the underlying mechanisms of myopia remain to be elucidated.

The current study had certain limitations. Firstly, the present study was limited by its cross-sectional design. Therefore, additional studies that include frequent follow-ups of these patients are warranted. Secondly, participants did not present with pathological myopia. Thus, further studies are needed.

In conclusion, a correlation between optic disc deformation and retinal vasculature in non-pathological highly myopic eyes was observed using OCTA. This association may explain the reduced peripapillary and macular vessel density in high myopia. According to the results of the present study, disc deformation (particularly optic disc tilt and $\beta$-PPA) may occur earlier than changes in the macular region in myopia retinopathy. Therefore, changes in the optic disc may be early signs of retinal changes in myopic eyes.

\section{Acknowledgements}

Not applicable.

\section{Funding}

The current study was supported by the Research Foundation of Beijing Friendship Hospital Affiliated to Capital Medical University, Beijing, China (grant no. yyqdkt2019-29).

\section{Availability of data and materials}

The datasets used and/or analyzed during the current study are available from the corresponding author on reasonable request.

\section{Authors' contributions}

YW contributed to the acquisition and analysis of data. JW designed the current study and revised the manuscript. JS designed the study, analyzed data and drafted the manuscript. All authors read and approved the final manuscript.

\section{Ethics approval and consent to participate}

The current study was approved by the Ethics Committee of the Beijing Friendship Hospital (Beijing, China). Written informed consent was obtained from all the examined patients and volunteering participants. 


\section{Patient consent for publication}

Not applicable.

\section{Competing interests}

The authors declare that they have no competing interests.

\section{References}

1. Jonas JB, Xu L, Wei WB, Wang XY, Jiang WJ, Bi HS and Jonas SP: Myopia in China: A population-based cross-sectional, histological, and experimental study. Lancet 388 (Suppl 1): S20, 2016.

2. Shimada N, Ohno-Matsui K, Harino S, Yoshida T, Yasuzumi K, Kojima A, Kobayashi K, Futagami S, Tokoro T and Mochizuki M: Reduction of retinal blood flow in high myopia. Graefes Arch Clin Exp Ophthalmol 242: 284-288, 2004.

3. La Spina C, Corvi F, Bandello F and Querques G: Static characteristics and dynamic functionality of retinal vessels in longer eyes with or without pathologic myopia. Graefes Arch Clin Exp Ophthalmol 254: 827-834, 2016

4. Li M, Yang Y, Jiang H, Giovanni G, Luiz R, Zheng F, Ke B, Qu DY and Wang JH: Retinal microvascular network and microcirculation assessments in high myopia. Am J Ophthalmol 174: 56-67, 2017.

5. Fan H, Chen HY, Ma HJ, Chang Z, Yin HQ, Ng DS, Cheung CY, Hu S, Xiang X, Tang SB and Li SN: Reduced macular vascular density in myopic eyes. Chin Med J (Engl) 130: 445-451, 2017.

6. Wang Q, Chan S, Yang JY, You B, Wang YX, Jonas JB and Wei WB: Vascular density in retina and choriocapillaris as measured by optical coherence tomography angiography. Am J Ophthalmol 168: 95-109, 2016

7. Samarawickrama C, Mitchell P, Tong L, Gazzard G, Lim L, Wong TY and Saw SM: Myopia-related optic disc and retinal changes in adolescent children from Singapore. Ophthalmology 118: 2050-2057, 2011.

8. Ohno-Matsui K: Proposed classification of posterior staphylomas based on analyses of eye shape by three-dimensiona magnetic resonance imaging and wide-field fundus imaging. Ophthalmology 121: 1798-1809, 2014.

9. Wang X, Kong X, Jiang C, Li M, Yu J and Sun X: Is the peripapillary retinal perfusion related to myopia in healthy eyes? A prospective comparative study. BMJ Open 6: e010791, 2016.

10. Sampson DM, Gong P, An D, Menghini M, Hansen A, Mackey DA, Sampson DD and Chen FK: Axial length variation impacts on superficial retinal vessel density and foveal avascular zone area measurements using optical coherence tomography angiography. Invest Ophthalmol Vis Sci 58: 3065-3072, 2017.
11. He J, Chen Q, Yin Y, Zhou H, Fan Y, Zhu JF, Zou HD and $\mathrm{Xu} \mathrm{X}$ : Association between retinal microvasculature and optic disc alterations in high myopia. Eye (Lond) 33: 1494-1503, 2019.

12. Ohno-Matsui K, Lai TY, Lai CC and Cheung CM: Updates of pathologic myopia. Prog Retin Eye Res 52: 156-187, 2016.

13. Tay E, Seah SK, Chan SP, Lim AT, Chew SJ, Foster PJ and Aung T: Optic disk ovality as an index of tilt and its relationship to myopia and perimetry. Am J Ophthalmol 139: 247-252, 2005.

14. Dai Y, Jonas JB, Huang H, Wang M and Sun X: Microstructure of parapapillary atrophy: Beta zone and gamma zone. Invest Ophthalmol Vis Sci 54: 2013-2018, 2013.

15. Guo Y, Liu LJ, Tang P, Feng Y, Lv YY, Wu M, Xu L and Jonas JB: Parapapillary gamma zone and progression of myopia in school children: The Beijing children eye study. Invest Ophthalmol Vis Sci 59: 1609-1616, 2018

16. Xu H, Deng G, Jiang C, Kong X, Yu J and Sun X: Microcirculatory responses to hyperoxia in macular and peripapillary regions. Invest Ophthalmol Vis Sci 57: 4464-4468, 2016.

17. Garg A, Blumberg DM, Al-Aswad LA, Oll M, Yzer S, Forbes M, Allikmets RL and Bearelly S: Associations between $\beta$-peripapillary atrophy and reticular pseudodrusen in early age-related macular degeneration. Invest Ophthalmol Vis Sci 58: 2810-2815, 2017

18. Kwon JW, Choi JA, Kim JS and La TY: Ganglion cell-inner plexiform layer, peripapillary retinal nerve fiber layer, and macular thickness in eyes with myopic $\beta$-zone parapapillary atrophy. J Ophthalmol 2016: 3746791, 2016.

19. Lee KM, Choung HK, Kim M, Oh S and Kim SH: Positional change of optic nerve head vasculature during axial elongation as evidence of lamina cribrosa shifting: Boramae myopia cohort study report 2. Ophthalmology 125: 1224-1233, 2018.

20. Zhang M, Hwang TS, Campbell JP, Bailey ST, Wilson DJ, Huang D and Jia Y: Projection-resolved optical coherence tomographic angiography. Biomed Opt Express 7: 816-828, 2016.

21. Landa G and Rosen RB: New patterns of retinal collateral circulation are exposed by a retinal functional imager (RFI). Br J Ophthalmol 94: 54-58, 2010.

22. Chui TY, Zhong Z and Burns SA: The relationship between peripapillary crescent and axial length: Implications for differential eye growth. Vision Res 51: 2132-2138, 2011.

23. Cai XB, Shen SR, Chen DF, Zhang Q and Jin ZB: An overview of myopia genetics. Exp Eye Res 188: 107778, 2019.

24. Bredrup C, Johansson S, Bindoff LA, Sztromwasser P, Kråkenes J Mellgren AE, Brurås KR, Lind O, Boman H, Knappskog PM and Rødahl E: High myopia-excavated optic disc anomaly associated with a frameshift mutation in the MYC-binding protein 2 gene (MYCBP2). Am J Ophthalmol 159: 973-979.e2, 2015. 